\title{
Association of Sleep Duration and Insomnia Symptoms with Components of Metabolic Syndrome and Inflammation in Middle-Aged and Older Adults with Metabolic Syndrome in Taiwan
}

\author{
Ahmad Syauqy ${ }^{1,2} \mathbb{D}$, Chien-Yeh Hsu ${ }^{3,4}$, Hsiao-Hsien Rau ${ }^{5}$, Adi Lukas Kurniawan ${ }^{1}$ and \\ Jane C-J Chao ${ }^{1,4,6, *}$ \\ 1 School of Nutrition and Health Sciences, College of Nutrition, Taipei Medical University, \\ 250 Wu-Hsing Street, Taipei 11031, Taiwan \\ 2 Department of Nutrition Science, Faculty of Medicine, Diponegoro University, Jl. Prof. H. Soedarto, S.H., \\ Tembalang, Semarang City, Central Java 50275, Indonesia \\ 3 Department of Information Management, National Taipei University of Nursing and Health Sciences, \\ 365 Ming-Te Road, Peitou District, Taipei 11219, Taiwan \\ 4 Master Program in Global Health and Development, College of Public Health, Taipei Medical University, \\ $250 \mathrm{Wu}$-Hsing Street, Taipei 11031, Taiwan \\ 5 Joint Commission of Taiwan, 31 Sec. 2 Sanmin Road, Banqiao District, New Taipei City 22069, Taiwan \\ 6 Nutrition Research Center, Taipei Medical University Hospital, 252 Wu-Hsing Street, Taipei 11031, Taiwan \\ * Correspondence: chenjui@tmu.edu.tw; Tel.: +886-2-2736-1661 (ext. 6548); Fax: +886-2-2736-3112
}

Received: 2 July 2019; Accepted: 7 August 2019; Published: 9 August 2019

check for

Abstract: The study determined the association of sleep duration and insomnia symptoms with the components of metabolic syndrome and inflammation in middle-aged and older adults with metabolic syndrome in Taiwan. This cross-sectional study used the database compiled in Taiwan between 2004-2013. A total of 26,016 volunteers aged 35 years and above were selected. Metabolic syndrome was defined according to the International Diabetes Federation. Compared with regular sleep duration (6-8 h/day), short ( $<6 \mathrm{~h} /$ day) or long sleep duration ( $>8 \mathrm{~h} /$ day) and insomnia symptoms significantly increased the odds ratios of high waist circumference, high blood pressure, low high-density lipoprotein-cholesterol, high triglycerides, high fasting blood glucose, and high C-reactive protein. Insomnia symptoms did not modify the effects of sleep duration on the components of metabolic syndrome and inflammation. Our study suggests that short or long sleep duration and insomnia symptoms may have an adverse effect on metabolic syndrome and inflammation.

Keywords: sleep duration; sleep quality; insomnia symptoms; metabolic syndrome; inflammation; cross-sectional study

\section{Introduction}

The International Diabetes Federation (IDF) stated that metabolic syndrome is characterized by central obesity, elevated blood pressure, dyslipidemia, and impaired blood glucose [1]. Metabolic syndrome elevated the risks of diabetes, cardiovascular diseases (CVD), and mortality [2,3]. A study revealed that individuals with metabolic syndrome tended to have inflammation and might deteriorate the risks of metabolic diseases and CVD [4]. The prevalence of metabolic syndrome has considerably increased in many countries [5-9]. A Nutrition and Health Survey in Taiwan (NAHSIT) between 1993-1996 and 2005-2008 documented that the prevalence of metabolic syndrome in Taiwan increased almost twofold from $13.6 \%$ to $25.5 \%$, respectively [10]. The IDF also declared that central obesity was the underlying factor of metabolic syndrome [1]. The prevalence of central obesity (waist circumference 
$>90 \mathrm{~cm}$ in men and $>80 \mathrm{~cm}$ in women) in Taiwan was $28.3 \%$ in men and $28.7 \%$ in women [11]. Therefore, the identification of modifiable risk factors may be crucial to prevent the development of metabolic syndrome.

Sleep, one of the most important factors, leads to several health-related issues [12]. Some studies revealed that there was a U-shaped relationship between sleep duration and adverse health outcomes [13-16]. Short sleep duration had negative effects on metabolic diseases such as obesity [13], hypertension [14], diabetes [15], and escalated mortality [16]. The association had also been shown in long sleep duration [13-16]. Although previous epidemiological studies reported that short or long sleep duration was related to metabolic syndrome and its components [17-22] as well as inflammation [23,24], none of the studies have investigated individuals with metabolic syndrome. Furthermore, insomnia, a public health issue, was related to sleep disorders. The overall prevalence of insomnia ranged from $8 \%$ to $40 \%$ worldwide [25]. However, the relationship between insomnia and metabolic syndrome remains unclear. Some previous studies demonstrated that insomnia was associated with metabolic syndrome [26,27], but some others did not [28,29]. Moreover, to our knowledge, no study has discussed the association of sleep duration and insomnia symptoms with the components of metabolic syndrome and inflammation using a population with metabolic syndrome. Therefore, further study is required in order to elucidate this association. The objective of the study was to determine the association of sleep duration and insomnia symptoms with the components of metabolic syndrome and an inflammatory marker in Taiwanese middle-aged and older adults with metabolic syndrome.

\section{Materials and Methods}

\subsection{Data Source and Volunteers}

We used a cross-sectional study, and the data were provided by a private health firm in Taiwan (MJ Health Management Institution in Taipei, Taoyuan, Taichung, and Kaohsiung) from 2004 to 2013. The institution collected personal demographic, medical, dietary, and lifestyle information by the self-administered questionnaires and provided medical examination such as anthropometric measurements, image examination, and biochemical tests of blood and urine from people who visited the MJ Health Screening Center in Taipei, Taoyuan, Taichung, and Kaohsiung for a regular health check-up. The questionnaires were available in Mandarin and English versions, and had been validated and standardized previously [30,31]. Details of the questionnaires have been published elsewhere [30,31]. All the volunteers filled in a consent form to allow the use of data without personal identification for research only. Detailed information and volunteers recruitment have been described elsewhere [32]. The committee of Taipei Medical University-Joint Institutional Review Board approved this study (TMU-JIRB N201706051). From the MJ database, 60,769 volunteers aged 35 years and above had metabolic syndrome. Of those, 23,377 volunteers who had chronic diseases such as liver disease, renal disorder, or cancer were excluded. After eliminating those with missing data $(n=11,376)$, the final sample size in our study was 26,016 volunteers.

\subsection{Definitions of Metabolic Syndrome and Inflammation}

Metabolic syndrome was characterized as volunteers with central obesity (waist circumference men $\geq 90 \mathrm{~cm}$; women $\geq 80 \mathrm{~cm}$ specifically for Asia/Taiwan) with two of the following factors: (1) high blood pressure (systolic blood pressure $\geq 130 \mathrm{mmHg}$, diastolic blood pressure $\geq 85 \mathrm{mmHg}$, or medication of formerly diagnosed hypertension), (2) low high-density lipoprotein-cholesterol (HDL-C) (men $<1.03 \mathrm{mmol} / \mathrm{L}$, women $<1.29 \mathrm{mmol} / \mathrm{L}$, or particular medication for dyslipidemia), (3) high triglycerides ( $\geq 1.70 \mathrm{mmol} / \mathrm{L}$ or particular medication for dyslipidemia), and (4) high fasting blood glucose (FBG) ( $\geq 5.60 \mathrm{mmol} / \mathrm{L}$ or formerly diagnosed type 2 diabetes) [1]. Inflammation was defined as high C-reactive protein (CRP) $(\geq 28.6 \mathrm{nmol} / \mathrm{L})[33]$. 


\subsection{Data Collection}

When visiting the MJ Health Screening Center for health check-up, volunteers recorded their sleep condition using a self-administered questionnaire. Volunteers filled the question of "How many hours do you usually sleep a day?" by one the following response options: <4 h, 4-6 h, 6-8 h, or $>8 \mathrm{~h}[31,34]$. In this study, we found only a small number of volunteers $(n=214,0.8 \%)$ who slept $<4 \mathrm{~h} /$ day; therefore, we pooled the data with those who slept for $4-6 \mathrm{~h} /$ day. To obtain the insomnia symptoms, the volunteers were asked, "How was your sleep condition in the last month?" with the following response options: difficulty initiating sleep, difficulty maintaining sleep, feeling of non-restorative sleep, use of sleeping pills, and sleeping well. According to the American Psychiatric Association [34,35], volunteers who responded to at least one of the former four options mentioned above were considered as having insomnia symptoms, while volunteers who responded that they were sleeping well were considered as not having insomnia symptoms.

Anthropometric and biochemical assessments were done by well-trained professionals at the MJ Health Screening Center and the MJ Central Laboratory, respectively. Volunteers were fasting for 12-14 h prior to blood drawn for biochemical measurements. Weight and height were assessed by an anthropometer (Nakamura KN-5000A, Tokyo, Japan). Body mass index (BMI) was calculated by the formula of $\mathrm{kg} / \mathrm{m}^{2}$. Waist circumference was assessed at the narrowest between the iliac crest and the bottom of the ribs. Blood pressure was measured by a sphygmomanometer (Citizen CH-5000, Tokyo, Japan) on the right arm. Plasma HDL-C, triglycerides, FBG, and CRP were assessed enzymatically using an auto-analyzer (Hitachi 7150, Tokyo, Japan) [31].

\subsection{Covariates}

We also collected some associated factors of metabolic syndrome including sex, age, marital status, level of education, current drinking and smoking status, physical activity, and diet using the self-administered questionnaire. Sex was dichotomized as men and women. Marital status was defined as never married, married, and divorced. Level of education was categorized as low (high school or below) and high (above high school). Current drinking and smoking status was dichotomized as no and yes. Physical activity was defined as low ( $<2 \mathrm{~h} /$ week) and high ( $\geq 2 \mathrm{~h} /$ week). Diet was evaluated using a food frequency questionnaire, and pointed to Taiwanese characteristics of dietary patterns as described previously [36,37]. In this study, data on the diet over the past month included milk, dairy products, eggs, meat, seafood, beans, vegetables, fruits, rice, whole grains, root crops, breads, instant noodles, and sugar. Then, we categorized the frequency of consumption into five groups: none or $<1$ serving/week, 1-3 servings/week, 4-6 servings/week, 1 serving/day, and $\geq 2$ servings/day.

\subsection{Statistical Analysis}

The data are presented as percentages for categorical variables or means \pm standard deviations (SD) for continuous variables. For categorical variables, a chi-squared test was performed to investigate the differences in the characteristics of the volunteers across sleep duration. For continuous variables, a general linear model test was conducted for comparison. The multivariable-adjusted logistic regression analysis was used to estimate the association of sleep duration and insomnia symptoms with the components of metabolic syndrome and CRP, and the odds ratios (OR) and 95\% confidence intervals (CIs) were determined. We conducted a stratified analysis by insomnia symptoms to evaluate the effect of insomnia symptoms on the association of sleep duration with the components of metabolic syndrome and CRP. All the regression models were controlled for sex (not including waist circumference and HDL-C), age, marital status, level of education, current drinking and smoking status, physical activity, diet, and insomnia symptoms or sleep duration. Volunteers who slept 6-8 h/day and without insomnia symptoms were selected as a reference group. A $p$ value $<0.05$ was considered statistically significant, and SPSS software (version 24, IBM Corp., Armonk, NY, USA) was used in this study. 


\section{Results}

Table 1 shows the characteristics of the volunteers across sleep duration. Among 26,016 volunteers (64.9\% men and 35.1\% women) with metabolic syndrome, $24.0 \%$ of volunteers slept $<6 \mathrm{~h} / \mathrm{day}, 66.7 \%$ slept 6-8 h/day, and $9.3 \%$ slept $>8 \mathrm{~h} /$ day. More than half of the volunteers $(n=14,261,54.8 \%)$ had insomnia symptoms, and $41.9 \%(n=10,901), 22.1 \%(n=5,750), 25.1 \%(n=6,530), 50.3 \%(n=13,086)$, and $70.1 \%(n=18,237)$ of volunteers had elevated systolic blood pressure, elevated diastolic blood pressure, low HDL-C, high triglycerides, and high FBG, respectively. Volunteers who slept $<6 \mathrm{~h} /$ day or $>8 \mathrm{~h} /$ day had significantly higher BMI $\left(27.3 \pm 2.6\right.$ and $27.2 \pm 2.6 \mathrm{~kg} / \mathrm{m}^{2}$ versus $\left.26.6 \pm 2.6 \mathrm{~kg} / \mathrm{m}^{2}\right)$, waist circumference $(89.9 \pm 8.1$ and $89.3 \pm 7.8 \mathrm{~cm}$ versus $88.8 \pm 8.7 \mathrm{~cm})$, systolic blood pressure $(124 \pm 41$ and $121 \pm 36 \mathrm{mmHg}$ versus $119 \pm 36 \mathrm{mmHg}$ ), diastolic blood pressure $(73 \pm 21$ and $72 \pm 23 \mathrm{mmHg}$ versus $71 \pm 21 \mathrm{mmHg})$, plasma triglycerides $(2.0 \pm 1.4$ and $2.0 \pm 1.2 \mathrm{mmol} / \mathrm{L}$ versus $1.9 \pm 1.3 \mathrm{mmol} / \mathrm{L})$, fasting blood glucose $(6.9 \pm 2.6$ and $6.3 \pm 1.8 \mathrm{mmol} / \mathrm{L}$ versus $6.2 \pm 1.7 \mathrm{mmol} / \mathrm{L})$, and CRP $(31.9 \pm 39.1$ and $29.7 \pm 36.9 \mathrm{nmol} / \mathrm{L}$ versus $27.7 \pm 33.0 \mathrm{nmol} / \mathrm{L}$ ) than those who slept $6-8 \mathrm{~h} /$ day. Dietary intake was different among the volunteers with different sleep duration, as shown in Table S1.

Table 1. Characteristics of the volunteers across sleep duration. ${ }^{1}$

\begin{tabular}{|c|c|c|c|c|}
\hline \multirow[t]{2}{*}{ Variables } & \multicolumn{3}{|c|}{ Sleep Duration (Hours/Day) } & \multirow[b]{2}{*}{$p$-Value } \\
\hline & $\begin{array}{c}<6 \\
(n=6262)\end{array}$ & $\begin{array}{c}6-8 \\
(n=17,356)\end{array}$ & $\begin{array}{c}>8 \\
(n=2398)\end{array}$ & \\
\hline Sex & & & & 0.045 \\
\hline Men (\%) & 64.6 & 64.8 & 66.8 & \\
\hline Women (\%) & 35.4 & 35.4 & 33.2 & \\
\hline Age (years) & $53.7 \pm 11.9$ & $53.5 \pm 11.8$ & $53.4 \pm 11.3$ & 0.464 \\
\hline \multicolumn{5}{|l|}{ Marital status } \\
\hline Not married & 3.7 & 3.1 & 2.6 & \multirow{3}{*}{0.000} \\
\hline Married & 83.2 & 84.5 & 87.4 & \\
\hline Divorced & 13.1 & 12.4 & 9.9 & \\
\hline \multicolumn{5}{|l|}{ Level of education } \\
\hline Low & 54.4 & 52.1 & 52.3 & \multirow[t]{2}{*}{0.005} \\
\hline High & 45.6 & 47.9 & 47.7 & \\
\hline \multicolumn{5}{|l|}{ Current drinking status } \\
\hline No & 95.4 & 96.0 & 91.5 & \multirow[t]{2}{*}{0.000} \\
\hline Yes & 4.6 & 4.0 & 8.5 & \\
\hline \multicolumn{5}{|l|}{ Current smoking status } \\
\hline No & 96.0 & 96.7 & 93.0 & \multirow[t]{2}{*}{0.000} \\
\hline Yes & 4.0 & 3.3 & 7.0 & \\
\hline \multicolumn{5}{|l|}{ Physical activity } \\
\hline Low & 48.4 & 46.2 & 41.8 & \multirow{3}{*}{0.000} \\
\hline Moderate & 45.5 & 47.8 & 52.2 & \\
\hline High & 6.1 & 6.0 & 6.0 & \\
\hline \multicolumn{5}{|l|}{ Insomnia symptoms } \\
\hline No & 50.6 & 38.6 & 78.7 & \multirow[t]{2}{*}{0.000} \\
\hline Yes & 49.4 & 61.4 & 21.3 & \\
\hline Body mass index $\left(\mathrm{kg} / \mathrm{m}^{2}\right)$ & $27.3 \pm 2.6$ & $26.6 \pm 2.6$ & $27.2 \pm 2.6$ & 0.000 \\
\hline Waist circumference $(\mathrm{cm})$ & $89.9 \pm 8.1$ & $88.8 \pm 8.7$ & $89.3 \pm 7.8$ & 0.000 \\
\hline Systolic BP (mmHg) & $124 \pm 41$ & $119 \pm 36$ & $121 \pm 36$ & 0.000 \\
\hline Diastolic BP (mmHg) & $73 \pm 21$ & $71 \pm 21$ & $72 \pm 23$ & 0.001 \\
\hline HDL-C (mmol/L) & $1.2 \pm 0.4$ & $1.3 \pm 0.4$ & $1.3 \pm 0.3$ & 0.001 \\
\hline Triglycerides (mmol/L) & $2.0 \pm 1.4$ & $1.9 \pm 1.3$ & $2.0 \pm 1.2$ & 0.003 \\
\hline FBG (mmol/L) & $6.9 \pm 2.6$ & $6.2 \pm 1.7$ & $6.3 \pm 1.8$ & 0.000 \\
\hline $\mathrm{CRP}$ (nmol/L) & $31.9 \pm 39.1$ & $27.7 \pm 33.0$ & $29.7 \pm 36.9$ & 0.001 \\
\hline
\end{tabular}

BP: blood pressure, HDL-C: high-density lipoprotein-cholesterol, FBG: fasting blood glucose, CRP: C-reactive protein. ${ }^{1}$ Data are presented as $\%$ for categorical variables and mean $\pm \mathrm{SD}$ for continuous variables.

The odds ratios of the components of metabolic syndrome and CRP across sleep duration are summarized in Table 2. There was a U-shaped relationship of sleep duration with the components of metabolic syndrome and CRP. Compared with the volunteers who slept 6-8 h/day, those who slept 
$<6 \mathrm{~h} /$ day had significantly increased odds ratios of high waist circumference, high blood pressure (except for high diastolic blood pressure in model 3), low HDL-C (except for low HDL-C in men in model 1), high triglycerides, high FBG, and high CRP in all models $(p<0.05)$. Meanwhile, volunteers who slept $>8 \mathrm{~h} /$ day had significantly increased odds ratios of high systolic blood pressure (only in model 3), low HDL-C in men, high triglycerides, and high FBG in all models $(p<0.05)$.

Table 2. Odds ratios $(95 \% \mathrm{CI})$ of the components of metabolic syndrome and C-reactive protein across sleep duration.

\begin{tabular}{|c|c|c|c|c|}
\hline \multirow[t]{2}{*}{ Variables } & \multicolumn{4}{|c|}{ Sleep Duration (Hours/Day) } \\
\hline & $<6$ & $6-8$ & $>8$ & $p$-Trend \\
\hline \multicolumn{5}{|c|}{$\begin{array}{l}\text { High waist circumference } \\
\text { (men) }\end{array}$} \\
\hline Model 11 & $1.303(1.061-1.600)$ & 1 & $1.040(0.991-1.091)$ & 0.014 \\
\hline Model $2^{2}$ & $1.236(1.005-1.520)$ & 1 & $1.019(0.971-1.070)$ & 0.027 \\
\hline Model $3^{3}$ & $1.246(0.979-1.513)$ & 1 & $1.030(0.981-1.081)$ & 0.044 \\
\hline \multicolumn{5}{|c|}{$\begin{array}{l}\text { High waist circumference } \\
\text { (women) }\end{array}$} \\
\hline Model 11 & $1.434(1.130-1.819)$ & 1 & $1.013(0.961-1.068)$ & 0.012 \\
\hline Model $2^{2}$ & 1.444 (1.139-1.833) & 1 & $1.010(0.958-1.065)$ & 0.009 \\
\hline Model $3^{3}$ & $1.410(1.112-1.788)$ & 1 & $1.009(0.957-1.065)$ & 0.015 \\
\hline \multicolumn{5}{|c|}{ High systolic BP } \\
\hline Model $1^{1}$ & $1.228(1.123-1.342)$ & 1 & $1.048(0.986-1.114)$ & 0.000 \\
\hline Model $2^{2}$ & $1.233(1.131-1.345)$ & 1 & $1.058(0.997-1.123)$ & 0.000 \\
\hline Model $3^{3}$ & $1.242(1.134-1.350)$ & 1 & 1.077 (1.017-1.077) & 0.000 \\
\hline \multicolumn{5}{|c|}{ High diastolic BP } \\
\hline Model $1^{1}$ & $1.326(1.076-1.578)$ & 1 & $1.011(0.896-1.141)$ & 0.031 \\
\hline Model $2^{2}$ & 1.309 (1.019-1.599) & 1 & $1.008(0.892-1.139)$ & 0.034 \\
\hline Model $3^{3}$ & $1.296(0.993-1.596)$ & 1 & $1.001(0.885-1.132)$ & 0.044 \\
\hline \multicolumn{5}{|c|}{ Low HDL-C (men) } \\
\hline Model $1^{1}$ & $1.120(0.949-1.321)$ & 1 & 1.089 (1.046-1.134) & 0.000 \\
\hline Model $2^{2}$ & $1.233(1.042-1.458)$ & 1 & $1.073(1.029-1.118)$ & 0.001 \\
\hline Model $3^{3}$ & $1.239(1.047-1.465)$ & 1 & $1.076(1.033-1.122)$ & 0.000 \\
\hline \multicolumn{5}{|c|}{ Low HDL-C (women) } \\
\hline Model $1^{1}$ & $1.106(1.057-1.156)$ & 1 & $1.005(0.839-1.203)$ & 0.000 \\
\hline Model $2^{2}$ & $1.055(1.005-1.104)$ & 1 & $1.026(0.855-1.231)$ & 0.044 \\
\hline Model $3^{3}$ & 1.061 (1.013-1.111) & 1 & $1.035(0.862-1.242)$ & 0.042 \\
\hline \multicolumn{5}{|c|}{ High triglycerides } \\
\hline Model $1^{1}$ & $1.180(1.047-1.129)$ & 1 & 1.096 (1.064-1.129) & 0.000 \\
\hline Model $2^{2}$ & $1.188(1.052-1.341)$ & 1 & $1.044(1.013-1.076)$ & 0.002 \\
\hline Model $3^{3}$ & $1.202(1.065-1.358)$ & 1 & $1.048(1.016-1.081)$ & 0.001 \\
\hline \multicolumn{5}{|l|}{ High FBG } \\
\hline Model $1^{1}$ & $1.270(1.105-1.459)$ & 1 & $1.044(1.010-1.078)$ & 0.000 \\
\hline Model $2^{2}$ & $1.316(1.146-1.511)$ & 1 & $1.041(1.008-1.075)$ & 0.000 \\
\hline Model $3^{3}$ & $1.304(1.136-1.498)$ & 1 & $1.038(1.005-1.072)$ & 0.000 \\
\hline \multicolumn{5}{|l|}{ High CRP } \\
\hline Model $1^{1}$ & $1.167(1.027-1.325)$ & 1 & 1.027 (0.994-1.061) & 0.029 \\
\hline Model 22 & $1.173(1.033-1.331)$ & 1 & $1.029(0.996-1.063)$ & 0.020 \\
\hline Model $3^{3}$ & 1.180 (1.039-1.339) & 1 & $1.029(0.996-1.063)$ & 0.017 \\
\hline
\end{tabular}

BP: blood pressure, HDL-C: high-density lipoprotein-cholesterol, FBG: fasting blood glucose, CRP: C-reactive protein. The odds ratios across categories of sleep duration were compared with the reference group (6-8 h/day). The variables were defined as high waist circumference (mean): $\geq 95.8 \mathrm{~cm}$ for men and $\geq 85.2 \mathrm{~cm}$ for women (because all the volunteers had central obesity (waist circumference $\geq 90 \mathrm{~cm}$ for men and $\geq 80 \mathrm{~cm}$ for women), mean waist circumference was used as the cutoff point), high systolic blood pressure: $\geq 130 \mathrm{mmHg}$, high diastolic blood pressure: $\geq 85 \mathrm{mmHg}$, low HDL-C: $<1.03 \mathrm{mmol} / \mathrm{L}$ for men and $<1.29 \mathrm{mmol} / \mathrm{L}$ for women, high triglycerides: $\geq 1.70 \mathrm{mmol} / \mathrm{L}$, high FBG: $\geq 5.60 \mathrm{mmol} / \mathrm{L}$, and high CRP: $\geq 28.6 \mathrm{nmol} / \mathrm{L}$. ${ }^{1}$ Unadjusted. ${ }^{2}$ Adjusted for sex (not including waist circumference and HDL-C), age, marital status, level of education, current drinking and smoking status, physical activity, and diet. ${ }^{3}$ Adjusted for sex (not for waist circumference and HDL-C), age, marital status, level of education, current drinking and smoking status, physical activity, diet, and insomnia symptoms. 
The odds ratios of the components of metabolic syndrome and CRP across volunteers with insomnia symptoms are presented in Table 3. Compared with the volunteers without insomnia symptoms, those with insomnia symptoms had significantly increased odds ratios of high waist circumference, high blood pressure (except for high systolic blood pressure in model 3), low HDL-C, high triglycerides, high FBG, and high CRP (only in model 1 ) in all the models $(p<0.05$ ).

Table 3. Odds ratios ( $95 \% \mathrm{CI}$ ) of the components of metabolic syndrome and C-reactive protein across volunteers with insomnia symptoms. OR: odds ratios.

\begin{tabular}{|c|c|c|}
\hline \multirow[t]{2}{*}{ Variables } & \multicolumn{2}{|c|}{ Volunteers with Insomnia Symptoms } \\
\hline & OR $(95 \%$ CI $)$ & $p$-Trend \\
\hline \multicolumn{3}{|c|}{ High waist circumference (men) } \\
\hline Model $1^{1}$ & $1.109(1.051-1.169)$ & 0.000 \\
\hline Model $2^{2}$ & $1.081(1.024-1.141)$ & 0.005 \\
\hline Model $3^{3}$ & $1.083(1.025-1.144)$ & 0.005 \\
\hline \multicolumn{3}{|c|}{$\begin{array}{l}\text { High waist circumference } \\
\text { (women) }\end{array}$} \\
\hline Model 1 & $1.130(1.040-1.227)$ & 0.004 \\
\hline Model 22 & $1.059(1.005-1.115)$ & 0.031 \\
\hline Model $3^{3}$ & 1.077 (1.004-1.155) & 0.038 \\
\hline \multicolumn{3}{|c|}{ High systolic BP } \\
\hline Model $1^{1}$ & $1.066(1.056-1.075)$ & 0.000 \\
\hline Model $2^{2}$ & $1.055(1.003-1.109)$ & 0.036 \\
\hline Model $3^{3}$ & $1.050(0.997-1.105)$ & 0.045 \\
\hline \multicolumn{3}{|c|}{ High diastolic BP } \\
\hline Model $1^{1}$ & $1.055(1.029-1.081)$ & 0.000 \\
\hline Model $2^{2}$ & $1.038(1.012-1.064)$ & 0.003 \\
\hline Model $3^{3}$ & $1.043(1.022-1.065)$ & 0.000 \\
\hline \multicolumn{3}{|c|}{ Low HDL-C (men) } \\
\hline Model $1^{1}$ & $1.092(1.052-1.132)$ & 0.000 \\
\hline Model 22 & $1.045(1.006-1.086)$ & 0.023 \\
\hline Model $3^{3}$ & $1.045(1.005-1.085)$ & 0.025 \\
\hline \multicolumn{3}{|c|}{ Low HDL-C (women) } \\
\hline Model $1^{1}$ & $1.106(1.066-1.147)$ & 0.000 \\
\hline Model $2^{2}$ & $1.050(1.012-1.091)$ & 0.011 \\
\hline Model $3^{3}$ & $1.050(1.011-1.090)$ & 0.012 \\
\hline \multicolumn{3}{|c|}{ High triglycerides } \\
\hline Model $1^{1}$ & $1.053(1.006-1.102)$ & 0.039 \\
\hline Model $2^{2}$ & $1.057(1.010-1.106)$ & 0.019 \\
\hline Model $3^{3}$ & $1.066(1.048-1.084)$ & 0.000 \\
\hline \multicolumn{3}{|l|}{ High FBG } \\
\hline Model 11 & $1.031(1.003-1.060)$ & 0.029 \\
\hline Model 22 & 1.035 (1.006-1.065) & 0.017 \\
\hline Model $3^{3}$ & $1.032(1.003-1.062)$ & 0.030 \\
\hline \multicolumn{3}{|l|}{ High CRP } \\
\hline Model 11 & $1.078(1.048-1.108)$ & 0.000 \\
\hline Model $2^{2}$ & $1.007(0.979-1.037)$ & 0.618 \\
\hline Model $3^{3}$ & $1.010(0.981-1.040)$ & 0.497 \\
\hline
\end{tabular}

BP: blood pressure, HDL-C: high-density lipoprotein-cholesterol, FBG: fasting blood glucose, CRP: C-reactive protein. The odds ratios across volunteers with insomnia symptoms were compared with the reference group (volunteers without insomnia symptoms). The variables were defined as high waist circumference (mean): $\geq 95.8 \mathrm{~cm}$ for men and $\geq 85.2 \mathrm{~cm}$ for women (because all the volunteers had central obesity (waist circumference $\geq 90 \mathrm{~cm}$ for men and $\geq 80 \mathrm{~cm}$ for women), mean waist circumference was used as the cutoff point), high systolic blood pressure: $\geq 130 \mathrm{mmHg}$, high diastolic blood pressure: $\geq 85 \mathrm{mmHg}$, low HDL-C: $<1.03 \mathrm{mmol} / \mathrm{L}$ for men and $<1.29 \mathrm{mmol} / \mathrm{L}$ for women, high triglycerides: $\geq 1.70 \mathrm{mmol} / \mathrm{L}$, high FBG: $\geq 5.60 \mathrm{mmol} / \mathrm{L}$, and high CRP: $\geq 28.6 \mathrm{nmol} / \mathrm{L}$. ${ }^{1}$ Unadjusted. ${ }^{2}$ Adjusted for sex (not for waist circumference and HDL-C), age, marital status, level of education, current drinking and smoking status, physical activity, and diet. ${ }^{3}$ Adjusted for sex (not including waist circumference and HDL-C), age, marital status, level of education, current drinking and smoking status, physical activity, diet, and sleep duration. 
We further used a stratified analysis to determine whether insomnia symptoms influenced the association of sleep duration with the components of metabolic syndrome and CRP, as shown in Table 4. The odds ratios for the components of metabolic syndrome and CRP also explained a U-shaped association with sleep duration in volunteers with or without insomnia symptoms. Compared with the volunteers who slept $6-8 \mathrm{~h} /$ day, those who slept $<6 \mathrm{~h} /$ day with or without insomnia symptoms had significantly increased odds ratios of high blood pressure, high FBG, and high CRP in all models $(p<0.05)$, and low HDL-C in model $1(p<0.05)$. Whereas the volunteers who slept $>8 \mathrm{~h} /$ day with or without insomnia symptoms had significantly increased odds ratios of high systolic blood pressure and high triglycerides in model 1 , as well as high CRP in model $2(p<0.05)$. Therefore, the association between sleep duration and metabolic syndrome was not modified by insomnia symptoms.

Table 4. Odds ratios (95\% CI) of the components of metabolic syndrome and C-reactive protein across volunteers with or without insomnia symptoms.

\begin{tabular}{|c|c|c|c|c|c|c|c|c|}
\hline \multirow[t]{3}{*}{ Variables } & \multicolumn{4}{|c|}{ Volunteers without Insomnia Symptoms } & \multicolumn{4}{|c|}{ Volunteers with Insomnia Symptoms } \\
\hline & \multicolumn{4}{|c|}{ Sleep Duration (Hours/Day) } & \multicolumn{4}{|c|}{ Sleep Duration (Hours/Day) } \\
\hline & $<6$ & 6-8 & $>8$ & $p$-Trend & $<6$ & $6-8$ & $>8$ & $p$-Trend \\
\hline \multicolumn{9}{|c|}{$\begin{array}{c}\text { High waist } \\
\text { circumference (men) }\end{array}$} \\
\hline Model 11 & $1.212(0.858-1.568)$ & 1 & $1.050(0.973-1.127)$ & 0.043 & $1.375(1.041-1.817)$ & 1 & $1.055(0.982-1.134)$ & 0.032 \\
\hline Model $2^{2}$ & $1.182(0.869-1.608)$ & 1 & $1.031(0.966-1.101)$ & 0.397 & $1.322(0.999-1.751)$ & 1 & $1.035(0.962-1.113)$ & 0.106 \\
\hline \multicolumn{9}{|c|}{$\begin{array}{c}\text { High waist } \\
\text { circumference (women) }\end{array}$} \\
\hline Model $1^{1}$ & $1.753(1.234-2.490)$ & 1 & $1.042(0.949-1.144)$ & 0.006 & $1.440(1.056-1.964)$ & 1 & $1.133(1.063-1.208)$ & 0.000 \\
\hline Model $2^{2}$ & $1.512(1.057-2.161)$ & 1 & $1.004(0.912-1.105)$ & 0.077 & $1.350(0.987-1.847)$ & 1 & $1.007(0.943-1.076)$ & 0.171 \\
\hline \multicolumn{9}{|c|}{ High systolic BP } \\
\hline Model $1^{1}$ & $1.423(1.157-1.742)$ & 1 & $1.111(1.029-1.199)$ & 0.000 & $1.634(1.367-1.954)$ & 1 & $1.096(1.014-1.184)$ & 0.000 \\
\hline Model $2^{2}$ & $1.439(1.167-1.770)$ & 1 & $1.085(1.003-1.173)$ & 0.000 & $1.650(1.375-1.981)$ & 1 & $1.070(0.988-1.158)$ & 0.000 \\
\hline \multicolumn{9}{|c|}{ High diastolic BP } \\
\hline Model $1^{1}$ & $1.203(1.071-1.352)$ & 1 & $1.065(0.956-1.187)$ & 0.007 & $1.160(1.082-1.244)$ & 1 & $1.102(1.034-1.174)$ & 0.000 \\
\hline Model $2^{2}$ & $1.197(1.065-1.346)$ & 1 & $1.063(0.953-1.185)$ & 0.009 & $1.153(1.075-1.236)$ & 1 & 1.102 (1.034-1.175) & 0.000 \\
\hline \multicolumn{9}{|c|}{ Low HDL-C (men) } \\
\hline Model $1^{1}$ & $1.242(0.858-1.627)$ & 1 & $1.066(0.989-1.143)$ & 0.035 & $1.387(1.075-1.790)$ & 1 & $1.006(0.963-1.052)$ & 0.042 \\
\hline Model $2^{2}$ & $1.243(0.931-1.658)$ & 1 & $1.006(0.968-1.046)$ & 0.218 & $1.360(1.054-1.756)$ & 1 & $1.005(0.962-1.051)$ & 0.061 \\
\hline \multicolumn{9}{|c|}{ Low HDL-C (women) } \\
\hline Model 11 & $1.078(1.021-1.139)$ & 1 & $1.033(0.808-1.320)$ & 0.025 & $1.183(1.090-1.284)$ & 1 & $1.013(0.775-1.325)$ & 0.000 \\
\hline Model $2^{2}$ & $1.020(0.965-1.079)$ & 1 & $1.011(0.789-1.295)$ & 0.779 & $1.142(1.085-1.199)$ & 1 & $1.067(0.842-1.292)$ & 0.807 \\
\hline \multicolumn{9}{|c|}{ High triglycerides } \\
\hline Model $1^{1}$ & $1.256(1.064-1.483)$ & 1 & 1.139 (1.086-1.195) & 0.000 & $1.105(0.929-1.314)$ & 1 & $1.058(1.019-1.100)$ & 0.012 \\
\hline Model $2^{2}$ & $1.364(1.151-1.617)$ & 1 & $1.129(1.075-1.186)$ & 0.000 & $1.110(0.931-1.324)$ & 1 & $1.002(0.963-1.043)$ & 0.008 \\
\hline \multicolumn{9}{|l|}{ High FBG } \\
\hline Model $1^{1}$ & $1.322(1.087-1.607)$ & 1 & $1.004(0.966-1.043)$ & 0.000 & $1.509(1.247-1.826)$ & 1 & $1.058(1.005-1.112)$ & 0.000 \\
\hline Model $2^{2}$ & $1.321(1.083-1.611)$ & 1 & $1.016(0.975-1.060)$ & 0.021 & $1.296(1.067-1.574)$ & 1 & 1.064 (1.011-1.121) & 0.006 \\
\hline \multicolumn{9}{|l|}{ High CRP } \\
\hline Model $1^{1}$ & $1.371(1.152-1.632)$ & 1 & $1.044(0.991-1.100)$ & 0.001 & $1.290(1.082-1.538)$ & 1 & $1.063(1.009-1.121)$ & 0.005 \\
\hline Model $2^{2}$ & $1.267(1.062-1.512)$ & 1 & $1.074(1.019-1.133)$ & 0.004 & $1.217(1.012-1.462)$ & 1 & $1.056(1.001-1.115)$ & 0.006 \\
\hline
\end{tabular}

BP: blood pressure, HDL-C: high-density lipoprotein-cholesterol, FBG: fasting blood glucose, CRP: C-reactive protein. The odds ratios across sleep duration were compared with the reference group ( $6-8 \mathrm{~h} /$ day). The variables were defined as high waist circumference (mean): $\geq 95.8 \mathrm{~cm}$ for men and $\geq 85.2 \mathrm{~cm}$ for women (because all the volunteers had central obesity (waist circumference $\geq 90 \mathrm{~cm}$ for men and $\geq 80 \mathrm{~cm}$ for women), mean waist circumference was used as the cutoff point), high systolic blood pressure: $\geq 130 \mathrm{mmHg}$, high diastolic blood pressure: $\geq 85 \mathrm{mmHg}$, low HDL-C: $<1.03 \mathrm{mmol} / \mathrm{L}$ for men and $<1.29 \mathrm{mmol} / \mathrm{L}$ for women, high triglycerides: $\geq 1.70 \mathrm{mmol} / \mathrm{L}$, high FBG: $\geq 5.60 \mathrm{mmol} / \mathrm{L}$, and high CRP: $\geq 28.6 \mathrm{nmol} / \mathrm{L} .{ }^{1}$ Unadjusted. ${ }^{2}$ Adjusted for sex (not for waist circumference and HDL-C), age, marital status, level of education, current drinking and smoking status, physical activity, and diet.

\section{Discussion}

The results were consistent with a U-shaped curvilinear association between sleep duration and the components of metabolic syndrome and inflammation. Short or long sleep duration and insomnia symptoms had an adverse effect on the components of metabolic syndrome and inflammation. Similarly, the previous epidemiological studies found that the U-shaped associations existed between sleep duration and metabolic diseases including obesity [38], hypertension [14], dyslipidemia [39], and diabetes [40]. In addition, the previous studies showed that short sleep duration was linked to metabolic syndrome [17-20] and inflammation [23]. Contrarily, few studies had found an association between 
long sleep duration and metabolic syndrome [17,18,21,22] and inflammation [24]. A meta-analysis study indicated that people who slept less than $6-7 \mathrm{~h}$ a day were at a higher risk of metabolic syndrome than those who slept between 7-8 h a day, and those who slept more than $8-9 \mathrm{~h}$ a day also showed an increased risk for metabolic syndrome [41]. They observed U-shaped associations between people who slept less than 6-7 h daily or more than 8-9 h daily, and the risk of metabolic syndrome as compared with those who slept between $7-8 \mathrm{~h}$ a day [41]. Our study found that after fully adjusting for confounding variables, the relationship between sleep duration and the components of metabolic syndrome and inflammation remained strong, suggesting that sleep duration constantly had an influence on the components of metabolic syndrome and inflammatory marker CRP.

There are several biologically plausible pathways that could potentially explain the association of short or long sleep duration with negative metabolic consequences. Short sleep duration may interfere with the homeostasis of some hormonal systems such as ghrelin and leptin. Short sleep duration or sleep restriction was associated with increased ghrelin and reduced leptin [13,42]. An increase in ghrelin and a decrease in leptin could lead to decreased energy expenditure, impaired glycemic control, increased appetite, and elevated caloric intake [13,42]; furthermore, increased energy consumption might further trigger obesity and the development of metabolic syndrome. Other potential mechanisms are that individuals who slept shorter could increase cytokines such as interleukin (IL)-6 and tumor necrosis factor (TNF)- $\alpha$ [43], elevate cortisol secretion, alter growth hormone metabolism [44], and activate the sympathetic nervous system [44]. These alterations may result in insulin resistance and obesity, which are the components of metabolic syndrome. Moreover, short sleep duration led to low-grade inflammation and elevated stress response in the hypothalamic-pituitary-adrenal axis [45,46], which then resulted in metabolic syndrome and CVD [47]. Contrarily, the mechanisms linked to the association between long sleep duration, metabolic syndrome, and inflammation are not clear yet. A previous study revealed that longer sleep duration ( $>9 \mathrm{~h} /$ day) was correlated with elevated CRP levels in women with type 2 diabetes $[24,48]$. High levels of pro-inflammatory cytokines were associated with increased sleep duration, and elevated CRP concentrations could promote the development of both metabolic disturbance and CVD [24]. Stranges et al. also found that long sleep duration $(>8 \mathrm{~h} /$ day) was positively correlated with physical inactivity compared with normal sleep duration (6-8 h/day), probably because individuals who slept longer had less time to do physical activity [48].

This study indicated that insomnia symptoms were significantly associated with the components of metabolic syndrome and CRP. Our study was comparable with other studies that indicated that insomnia symptoms-including difficulty falling asleep, difficulty maintaining sleep, and experiencing unrefreshing sleep-were significantly correlated with metabolic syndrome [26,27]. A meta-analysis of epidemiological studies concluded that depressive symptoms, fatigue, and sleep fragmentation were considered as residual confounding factors, and comorbidity might accelerate the development of metabolic syndrome [49]. An epidemiological study also revealed that metabolic syndrome was closely related to insomnia and other sleep disorders [50]. Moreover, the evidence suggests that individuals with insomnia had increased pro-inflammatory cytokines such as IL-6, nuclear factor- $\mathrm{kB}$, and TNF- $\alpha[51,52]$. This study showed that the association between sleep duration and metabolic syndrome was not changed by insomnia symptoms after adjusting for confounding variables, indicating that insomnia symptoms consistently affected the components of metabolic syndrome.

The mechanism of the relationship of insomnia symptoms with metabolic syndrome and inflammation is not fully understood yet. The hyperactivity of the hypothalamic-pituitary-adrenal axis responding to insomnia could lead to metabolic syndrome [53]. Additionally, insulin resistance may be induced by sleep disorder, including sleep fragmentation or sleep restriction [54,55], which had a significant impact on the development of metabolic syndrome [56]. Accordingly, prolonged sleep deprivation could regulate the impact of glucose metabolism and further develop metabolic syndrome [57]. Our stratified analysis revealed that insomnia symptoms did not significantly influence the association of sleep duration with the components of metabolic syndrome and CRP as reported in the 
previous study [34], suggesting that there were no differences in the association of sleep duration with metabolic syndrome and inflammation between the individuals with and without insomnia symptoms.

This study has some important strengths. To the best of our knowledge, this was the first study to discuss the association of sleep duration and insomnia symptoms with the components of metabolic syndrome and inflammatory markers in Taiwanese aged 35 years and over with metabolic syndrome. We used the IDF definition to classify the components of metabolic syndrome, which included central obesity as a key component [1]. The prevalence of metabolic syndrome or central obesity has been escalating among Taiwanese aged 35 years and over [10,11]. Therefore, we used the IDF definition to reflect the most prevalent health issues in Taiwan. Moreover, our study had a large sample size from the population of interest. However, this study had certain limitations. First, similar to most other epidemiological studies, we used a self-reported questionnaire to examine sleep duration instead of an objective measurement of sleep using actigraphic or polysomnographic measurement, which was not practical in a large-scale study. Nonetheless, the previous studies found that both self-reported and objective measurements of sleep quality were correlated with an increased risk of metabolic diseases [58,59]. In addition, we did not evaluate sleep apnea, which might have an effect on the association of sleep duration with metabolic syndrome and inflammation, as the individuals often complained of insomnia symptoms such as difficulties falling asleep and frequent awakenings [60]. However, we adjusted for some confounding variables including sex, age, marital status, level of education, current drinking and smoking status, physical activity, and diet. Finally, the cross-sectional study could not specify causality between the variables. Future studies are needed to clarify the mechanisms underlying the association between sleep duration, insomnia symptoms, metabolic syndrome, and inflammation.

\section{Conclusions}

Our study suggests that short or long sleep duration and insomnia symptoms have an adverse effect on metabolic syndrome and inflammation.

Supplementary Materials: The following is available online at http://www.mdpi.com/2072-6643/11/8/1848/s1, Table S1. Dietary intake of the volunteers across sleep duration.

Author Contributions: A.S. and J.C.-J.C. designed and conducted the research; C.-Y.H. and H.-H.R. managed and retrieved the data; A.S., J.C.-J.C., C.-Y.H., A.L.K., and H.-H.R. contributed to data analysis and interpretation of data; A.S. and J.C.-J.C. wrote the manuscript. All authors participated in critical revision of the manuscript for important intellectual content and have read and approved the final manuscript.

Funding: This research received no external funding.

Conflicts of Interest: The authors declare no conflict of interest.

\section{References}

1. Alberti, K.G.M.; Zimmet, P.; Shaw, J. The metabolic syndrome-a new worldwide definition. Lancet 2005, 366, 1059-1062. [CrossRef]

2. Wilson, P.W.; D'Agostino, R.B.; Parise, H.; Sullivan, L.; Meigs, J.B. Metabolic syndrome as a precursor of cardiovascular disease and type 2 diabetes mellitus. Circulation 2005, 112, 3066-3072. [CrossRef] [PubMed]

3. Gami, A.S.; Witt, B.J.; Howard, D.E.; Erwin, P.J.; Gami, L.A.; Somers, V.K.; Montori, V.M. Metabolic syndrome and risk of incident cardiovascular events and death: A systematic review and meta-analysis of longitudinal studies. J. Am. Coll. Cardiol. 2007, 49, 403-414. [CrossRef] [PubMed]

4. Ridker, P.M.; Buring, J.E.; Cook, N.R.; Rifai, N. C-reactive protein, the metabolic syndrome, and risk of incident cardiovascular events: An 8-year follow-up of 14719 initially healthy American women. Circulation 2003, 107, 391-397. [CrossRef] [PubMed]

5. Gundogan, K.; Bayram, F.; Capak, M.; Tanriverdi, F.; Karaman, A.; Ozturk, A.; Altunbas, H.; Gokce, C.; Kalkan, A.; Yazici, C. Prevalence of metabolic syndrome in the Mediterranean region of Turkey: Evaluation of hypertension, diabetes mellitus, obesity and dyslipidemia. Metab. Syndr. Relat. Disord. 2009, 7, 427-434. [CrossRef] [PubMed] 
6. Puenpatom, R.A.; Victor, T.W. Increased prevalence of metabolic syndrome in individuals with osteoarthritis: An analysis of NHANES III data. Postgrad. Med. 2009, 121, 9-20. [CrossRef] [PubMed]

7. Beltran-Sanchez, H.; Harhay, M.O.; Harhay, M.M.; McElligott, S. Prevalence and trends of metabolic syndrome in the adult U.S. population, 1999-2010. J. Am. Coll. Cardiol. 2013, 62, 697-703. [CrossRef] [PubMed]

8. Scuteri, A.; Laurent, S.; Cucca, F.; Cockcroft, J.; Cunha, P.G.; Manas, L.R.; Mattace Raso, F.U.; Muiesan, M.L.; Ryliskyte, L.; Rietzschel, E.; et al. Metabolic syndrome across Europe: Different clusters of risk factors. Eur. J. Prev. Cardiol. 2015, 22, 486-491. [CrossRef] [PubMed]

9. Lim, K.G.; Cheah, W.K. A review of metabolic syndrome research in Malaysia. Med. J. Malaysia 2016, 71, 20-28.

10. Yeh, C.J.; Chang, H.Y.; Pan, W.H. Time trend of obesity, the metabolic syndrome and related dietary pattern in Taiwan: From NAHSIT 1993-1996 to NAHSIT 2005-2008. Asia Pac. J. Clin. Nutr. 2011, 20, 292-300.

11. Hwang, L.C.; Bai, C.H.; Chen, C.J. Prevalence of obesity and metabolic syndrome in Taiwan. J. Formos. Med. Assoc. 2006, 105, 626-635. [CrossRef]

12. Covassin, N.; Singh, P. Sleep duration and cardiovascular disease risk: Epidemiologic and experimental evidence. Sleep Med. Clin. 2016, 11, 81-89. [CrossRef] [PubMed]

13. Taheri, S.; Lin, L.; Austin, D.; Young, T.; Mignot, E. Short sleep duration is associated with reduced leptin, elevated ghrelin, and increased body mass index. PLoS Med. 2004, 1, e62. [CrossRef] [PubMed]

14. Gottlieb, D.J.; Redline, S.; Nieto, F.J.; Baldwin, C.M.; Newman, A.B.; Resnick, H.E.; Punjabi, N.M. Association of usual sleep duration with hypertension: The Sleep Heart Health Study. Sleep 2006, 29, 1009-1014. [CrossRef] [PubMed]

15. Gottlieb, D.J.; Punjabi, N.M.; Newman, A.B.; Resnick, H.E.; Redline, S.; Baldwin, C.M.; Nieto, F.J. Association of sleep time with diabetes mellitus and impaired glucose tolerance. Arch. Intern. Med. 2005, 165, 863-867. [CrossRef]

16. Patel, S.R.; Ayas, N.T.; Malhotra, M.R.; White, D.P.; Schernhammer, E.S.; Speizer, F.E.; Stampfer, M.J.; Hu, F.B. A prospective study of sleep duration and mortality risk in women. Sleep 2004, 27, 440-444. [CrossRef] [PubMed]

17. Choi, K.M.; Lee, J.S.; Park, H.S.; Baik, S.H.; Choi, D.S.; Kim, S.M. Relationship between sleep duration and the metabolic syndrome: Korean National Health and Nutrition Survey 2001. Int. J. Obes. (Lond.) 2008, 32, 1091-1097. [CrossRef] [PubMed]

18. Hall, M.H.; Muldoon, M.F.; Jennings, J.R.; Buysse, D.J.; Flory, J.D.; Manuck, S.B. Self-reported sleep duration is associated with the metabolic syndrome in midlife adults. Sleep 2008, 31, 635-643. [CrossRef]

19. Katano, S.; Nakamura, Y.; Nakamura, A.; Murakami, Y.; Tanaka, T.; Takebayashi, T.; Okayama, A.; Miura, K.; Okamura, T.; Ueshima, H. Relationship between sleep duration and clustering of metabolic syndrome diagnostic components. Diabetes Metab. Syndr. Obes. 2011, 4, 119-125. [CrossRef]

20. Kobayashi, D.; Takahashi, O.; Deshpande, G.A.; Shimbo, T.; Fukui, T. Relation between metabolic syndrome and sleep duration in Japan: A large scale cross-sectional study. Intern. Med. 2011, 50, 103-107. [CrossRef]

21. Arora, T.; Jiang, C.Q.; Thomas, G.N.; Lam, K.B.; Zhang, W.S.; Cheng, K.K.; Lam, T.H.; Taheri, S. Self-reported long total sleep duration is associated with metabolic syndrome: The Guangzhou Biobank Cohort Study. Diabetes Care 2011, 34, 2317-2319. [CrossRef] [PubMed]

22. Santos, A.C.; Ebrahim, S.; Barros, H. Alcohol intake, smoking, sleeping hours, physical activity and the metabolic syndrome. Prev. Med. 2007, 44, 328-334. [CrossRef] [PubMed]

23. Fukuoka, R.; Kohno, T.; Kohsaka, S.; Yanagisawa, R.; Kawakami, T.; Hayashida, K.; Kanazawa, H.; Yuasa, S.; Maekawa, Y.; Sano, M.; et al. Nocturnal intermittent hypoxia and short sleep duration are independently associated with elevated C-reactive protein levels in patients with coronary artery disease. Sleep Med. 2017, 29, 29-34. [CrossRef] [PubMed]

24. Williams, C.J.; Hu, F.B.; Patel, S.R.; Mantzoros, C.S. Sleep duration and snoring in relation to biomarkers of cardiovascular disease risk among women with type 2 diabetes. Diabetes Care 2007, 30, 1233-1240. [CrossRef] [PubMed]

25. Vgontzas, A.N.; Fernandez-Mendoza, J. Insomnia with short sleep duration: Nosological, diagnostic and treatment implications. Sleep Med. Clin. 2013, 8, 309-322. [CrossRef] [PubMed]

26. Chen, L.J.; Lai, Y.J.; Sun, W.J.; Fox, K.R.; Chu, D.; Ku, P.W. Associations of exercise, sedentary time and insomnia with metabolic syndrome in Taiwanese older adults: A 1-year follow-up study. Endocr. Res. 2015, 40, 220-226. [CrossRef] 
27. Lin, S.C.; Sun, C.A.; You, S.L.; Hwang, L.C.; Liang, C.Y.; Yang, T.; Bai, C.H.; Chen, C.H.; Wei, C.Y.; Chou, Y.C. The link of self-reported insomnia symptoms and sleep duration with metabolic syndrome: A Chinese population-based study. Sleep 2016, 39, 1261-1266. [CrossRef]

28. Troxel, W.M.; Buysse, D.J.; Matthews, K.A.; Kip, K.E.; Strollo, P.J.; Hall, M.; Drumheller, O.; Reis, S.E. Sleep symptoms predict the development of the metabolic syndrome. Sleep 2010, 33, 1633-1640. [CrossRef]

29. Chedraui, P.; San Miguel, G.; Villacreses, D.; Dominguez, A.; Jaramillo, W.; Escobar, G.S.; Pérez-López, F.R.; Genazzani, A.R.; Simoncini, T. Assessment of insomnia and related risk factors in postmenopausal women screened for the metabolic syndrome. Maturitas 2013, 74, 154-159. [CrossRef]

30. Wen, C.P.; Wai, J.P.; Tsai, M.K.; Yang, Y.C.; Cheng, T.Y.; Lee, M.C.; Chan, H.T.; Tsao, C.K.; Tsai, S.P.; Wu, X. Minimum amount of physical activity for reduced mortality and extended life expectancy: A prospective cohort study. Lancet 2011, 378, 1244-1253. [CrossRef]

31. Lao, X.Q.; Deng, H.B.; Liu, X.; Chan, T.C.; Zhang, Z.; Chang, L.Y.; Yeoh, E.K.; Tam, T.; Wong, M.C.S.; Thomas, G.N. Increased leisure-time physical activity associated with lower onset of diabetes in 44828 adults with impaired fasting glucose: A population-based prospective cohort study. Br. J. Sports Med. 2019, 53, 895-900. [CrossRef] [PubMed]

32. Wen, C.P.; Cheng, T.Y.D.; Tsai, M.K.; Chang, Y.C.; Chan, H.T.; Tsai, S.P.; Chiang, P.H.; Hsu, C.C.; Sung, P.K.; Hsu, Y.H.; et al. All-cause mortality attributable to chronic kidney disease: A prospective cohort study based on 462293 adults in Taiwan. Lancet 2008, 371, 2173-2182. [CrossRef]

33. Pearson, T.A.; Mensah, G.A.; Alexander, R.W.; Anderson, J.L.; Cannon, R.O., 3rd; Criqui, M.; Fadl, Y.Y.; Fortmann, S.P.; Hong, Y.; Myers, G.L.; et al. Markers of inflammation and cardiovascular disease: Application to clinical and public health practice: A statement for healthcare professionals from the centers for disease control and prevention and the American Heart Association. Circulation 2003, 107, 499-511. [CrossRef] [PubMed]

34. Deng, H.B.; Tam, T.; Zee, B.C.; Chung, R.Y.; Su, X.; Jin, L.; Chan, T.C.; Chang, L.Y.; Yeoh, E.K.; Lao, X.Q. Short sleep duration increases metabolic impact in healthy adults: A population-based cohort study. Sleep 2017, 40. [CrossRef] [PubMed]

35. American Psychiatric Association; DSM-5 Task Force. Diagnostic and Statistical Manual of Mental Disorders (DSM-5), 5th ed.; American Psychiatric Publishing, Inc.: Arlington, VA, USA, 2013.

36. Syauqy, A.; Hsu, C.Y.; Rau, H.H.; Chao, J.C.J. Association of dietary patterns with components of metabolic syndrome and inflammation among middle-aged and older adults with metabolic syndrome in Taiwan. Nutrients 2018, 10, 143. [CrossRef] [PubMed]

37. Syauqy, A.; Hsu, C.Y.; Rau, H.H.; Chao, J.C.J. Association of dietary patterns, anthropometric measurements and metabolic parameters with C-reactive protein and neutrophil-to-lymphocyte ratio in middle-aged and older adults with metabolic syndrome in Taiwan: A cross-sectional study. Nutr. J. 2018, 17, 106. [CrossRef] [PubMed]

38. Lin, C.L.; Lin, C.P.; Chen, S.W.; Wu, H.C.; Tsai, Y.H. The association between sleep duration and overweight or obesity in Taiwanese adults: A cross-sectional study. Obes. Res. Clin. Pract. 2018, 12, 384-388. [CrossRef]

39. Lin, P.M.D.; Chang, K.T.; Lin, Y.A.; Tzeng, I.S.; Chuang, H.H.; Chen, J.Y. Association between self-reported sleep duration and serum lipid profile in a middle-aged and elderly population in Taiwan: A community-based, cross-sectional study. BMJ Open 2017, 7, e015964. [CrossRef]

40. Yaggi, H.K.; Araujo, A.B.; McKinlay, J.B. Sleep duration as a risk factor for the development of type 2 diabetes. Diabetes Care 2006, 29, 657-661. [CrossRef]

41. Ju, S.Y.; Choi, W.S. Sleep duration and metabolic syndrome in adult populations: A meta-analysis of observational studies. Nutr. Diabetes 2013, 3, e65. [CrossRef]

42. Spiegel, K.; Tasali, E.; Penev, P.; Van Cauter, E. Brief communication: Sleep curtailment in healthy young men is associated with decreased leptin levels, elevated ghrelin levels and increased hunger and appetite. Ann. Intern. Med. 2004, 141, 846-850. [CrossRef]

43. Vgontzas, A.N.; Zoumakis, E.; Bixler, E.O.; Lin, H.M.; Follett, H.; Kales, A.; Chrousos, G.P. Adverse effects of modest sleep restriction on sleepiness, performance, and inflammatory cytokines. J. Clin. Endocrinol. Metab. 2004, 89, 2119-2126. [CrossRef]

44. Spiegel, K.; Leproult, R.; Van Cauter, E. Impact of sleep debt on metabolic and endocrine function. Lancet 1999, 354, 1435-1439. [CrossRef] 
45. Buckley, T.M.; Schatzberg, A.F. On the interactions of the hypothalamic-pituitary-adrenal (HPA) axis and sleep: Normal HPA axis activity and circadian rhythm, exemplary sleep disorders. J. Clin. Endocrinol. Metab. 2005, 90, 3106-3114. [CrossRef] [PubMed]

46. de Rooij, S.R.; Nijpels, G.; Nilsson, P.M.; Nolan, J.J.; Gabriel, R.; Bobbioni-Harsch, E.; Mingrone, G.; Dekker, J.M. Low-grade chronic inflammation in the relationship between insulin sensitivity and cardiovascular disease (RISC) population: Associations with insulin resistance and cardiometabolic risk profile. Diabetes Care 2009, 32, 1295-1301. [CrossRef] [PubMed]

47. Meier-Ewert, H.K.; Ridker, P.M.; Rifai, N.; Regan, M.M.; Price, N.J.; Dinges, D.F.; Mullington, J.M. Effect of sleep loss on C-reactive protein, an inflammatory marker of cardiovascular risk. J. Am. Coll. Cardiol. 2004, 43, 678-683. [CrossRef]

48. Stranges, S.; Dorn, J.M.; Shipley, M.J.; Kandala, N.B.; Trevisan, M.; Miller, M.A.; Donahue, R.P.; Hovey, K.M.; Ferrie, J.E.; Marmot, M.G.; et al. Correlates of short and long sleep duration: A cross-cultural comparison between the United Kingdom and the United States: The Whitehall II Study and the Western New York Health Study. Am. J. Epidemiol. 2008, 168, 1353-1364. [CrossRef]

49. Pan, A.; Keum, N.; Okereke, O.I.; Sun, Q.; Kivimaki, M.; Rubin, R.R.; Hu, F.B. Bidirectional association between depression and metabolic syndrome: A systematic review and meta-analysis of epidemiological studies. Diabetes Care 2012, 35, 1171-1180. [CrossRef]

50. Ikeda, M.; Kaneita, Y.; Uchiyama, M.; Mishima, K.; Uchimura, N.; Nakaji, S.; Akashiba, T.; Itani, O.; Aono, H.; Ohida, T. Epidemiological study of the associations between sleep complaints and metabolic syndrome in Japan. Sleep Biol. Rhythms. 2014, 12, 269-278. [CrossRef]

51. Vgontzas, A.N.; Chrousos, G.P. Sleep, the hypothalamic-pituitary-adrenal axis, and cytokines: Multiple interactions and disturbances in sleep disorders. Endocrinol. Metab. Clin. N. Am. 2002, 31, 15-36. [CrossRef]

52. Irwin, M.R.; Wang, M.; Ribeiro, D.; Cho, H.J.; Olmstead, R.; Breen, E.C.; Martinez-Maza, O.; Cole, S. Sleep loss activates cellular inflammatory signaling. Biol. Psychiatry 2008, 64, 538-540. [CrossRef] [PubMed]

53. Cagampang, F.R.; Poore, K.R.; Hanson, M.A. Developmental origins of the metabolic syndrome: Body clocks and stress responses. Brain Behav. Immun. 2011, 25, 214-220. [CrossRef] [PubMed]

54. Buxton, O.M.; Pavlova, M.; Reid, E.W.; Wang, W.; Simonson, D.C.; Adler, G.K. Sleep restriction for 1 week reduces insulin sensitivity in healthy men. Diabetes 2010, 59, 2126-2133. [CrossRef] [PubMed]

55. Stamatakis, K.A.; Punjabi, N.M. Effects of sleep fragmentation on glucose metabolism in normal subjects. Chest 2010, 137, 95-101. [CrossRef] [PubMed]

56. Cornier, M.A.; Dabelea, D.; Hernandez, T.L.; Lindstrom, R.C.; Steig, A.J.; Stob, N.R.; Van Pelt, R.E.; Wang, H.; Eckel, R.H. The metabolic syndrome. Endocr. Rev. 2008, 29, 777-822. [CrossRef] [PubMed]

57. Wang, Y.; Jiang, T.; Wang, X.; Zhao, J.; Kang, J.; Chen, M.; Wang, H.; Niu, L.; Wang, Y.; Zhou, Y.; et al. Association between insomnia and metabolic syndrome in a Chinese Han population: A cross-sectional study. Sci. Rep. 2017, 7, 10893. [CrossRef] [PubMed]

58. Lauderdale, D.S.; Knutson, K.L.; Yan, L.L.; Liu, K.; Rathouz, P.J. Self-reported and measured sleep duration: How similar are they? Epidemiology 2008, 19, 838-845. [CrossRef] [PubMed]

59. Silva, G.E.; Goodwin, J.L.; Sherrill, D.L.; Arnold, J.L.; Bootzin, R.R.; Smith, T.; Walsleben, J.A.; Baldwin, C.M.; Quan, S.F. Relationship between reported and measured sleep times: The Sleep Heart Health Study (SHHS). J. Clin. Sleep Med. 2007, 3, 622-630. [PubMed]

60. Beneto, A.; Gomez-Siurana, E.; Rubio-Sanchez, P. Comorbidity between sleep apnea and insomnia. Sleep Med. Rev. 2009, 13, 287-293. [CrossRef]

(C) 2019 by the authors. Licensee MDPI, Basel, Switzerland. This article is an open access article distributed under the terms and conditions of the Creative Commons Attribution (CC BY) license (http://creativecommons.org/licenses/by/4.0/). 